\title{
Perioperative Dexmedetomidine Improves Outcomes of Kidney Transplant
}

\author{
Jun Chen, MD'1, Fuhai Ji, MD, PhD ${ }^{1}$, Zhongmin $\mathrm{Li}^{2}$ and Hong $\mathrm{Liu}^{3}$ \\ ${ }^{1}$ Department of Anesthesiology, the First Affiliated Hospital of Soochow University; \\ ${ }^{2}$ Department of Medicine and ${ }^{3}$ Department of Anesthesiology and Pain Medicine, \\ University of California Davis Health
}

\begin{abstract}
Background: Graft function is crucial for successful kidney transplantation. Many factors may affect graft function or cause delayed graft function (DGF), which decreases the prognosis for graft survival. Dexmedetomidine has been shown to be renal protective in both pre-clinical and clinical studies. This study was designed to evaluate whether the perioperative use of dexmedetomidine could improve the incidence of function of graft kidney and complications after kidney transplantation.
\end{abstract}

Materials and methods: This study was a singlecenter, retrospective cohort study involving 797 consecutive patients who underwent renal transplantation at a university medical center from January 1, 2012, to July 22, 2014. A total of 780 patients met the inclusion criteria. 315 received intravenous dexmedetomidine infusion during surgery and 465 did not. The primary outcomes are major adverse complications included delayed graft function (DGF) and acute rejection in the early post transplantation phase. The secondary outcomes included length of hospital stay (LOS), infection, overall complication, graft functional status, post transplantation serum creatinine ( $\mathrm{SCr}$ ) values and estimate glomerular filtration rate (eGFR).

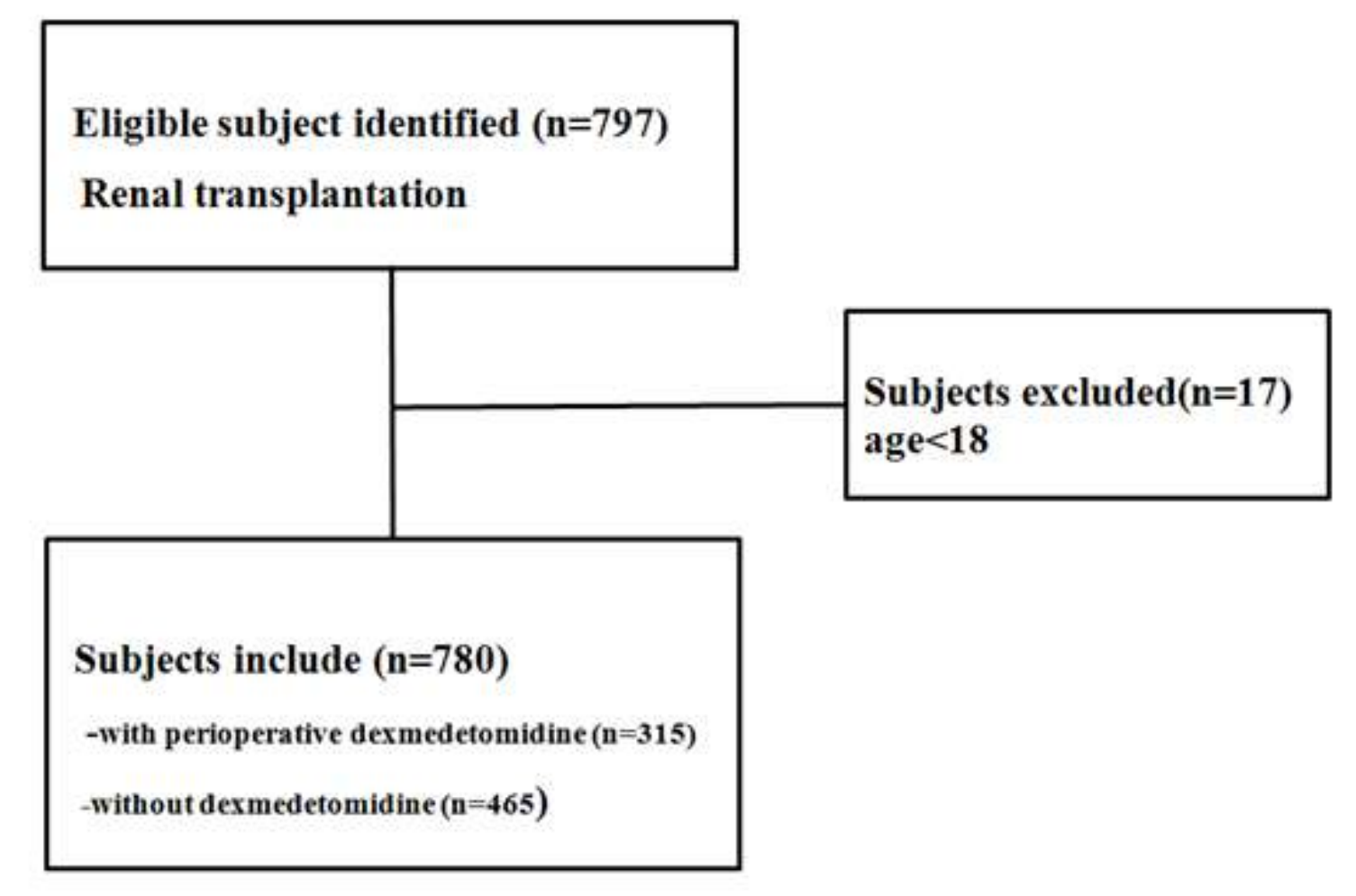

Figure 1: Study population recruitment summary.

Results: Dexmedetomidine use significantly reduced morbidity of DGF (19.37\% vs. $23.66 \%$; adjusted OR, $0.744 ; 95 \% \mathrm{CI}, 0.564-0.981 ; \mathrm{P}=0.036$ ), risk of infection $(8.25 \%$ vs. $15.70 \%$, adjusted OR, 0.489; $95 \% \mathrm{CI} ; 0.352-0.678, \mathrm{P}<0.0001)$, risk of acute rejection $(1.27 \%$ vs. $2.58 \%$; adjusted OR, 0.401 ; $95 \% \mathrm{CI}, 0.182-0.887 ; \mathrm{p}=0.024)$ in the early posttransplantation phase.

Sources: This work was supported by the Department of Anesthesiology and Pain Medicine, Department of Internal Medicine of University of California Davis Health System and NIH grant UL1 TR000002 of the University of California Davis Health
Perioperative dexmedetomidine therapy reduced the risk of overall complications (26.67\% vs.36.34\%; adjusted OR, 0.638; 95\% CI, $0.509-0.799 ; \mathrm{P}<0.0001)$ and LOS (6.4 vs. 7.1; $\mathrm{P}<0.0001)$. Use of dexmedetomidine has no marked impact on post transplantation 7 day, 30 day and 90 day $\mathrm{SCr}$ and eGFR.

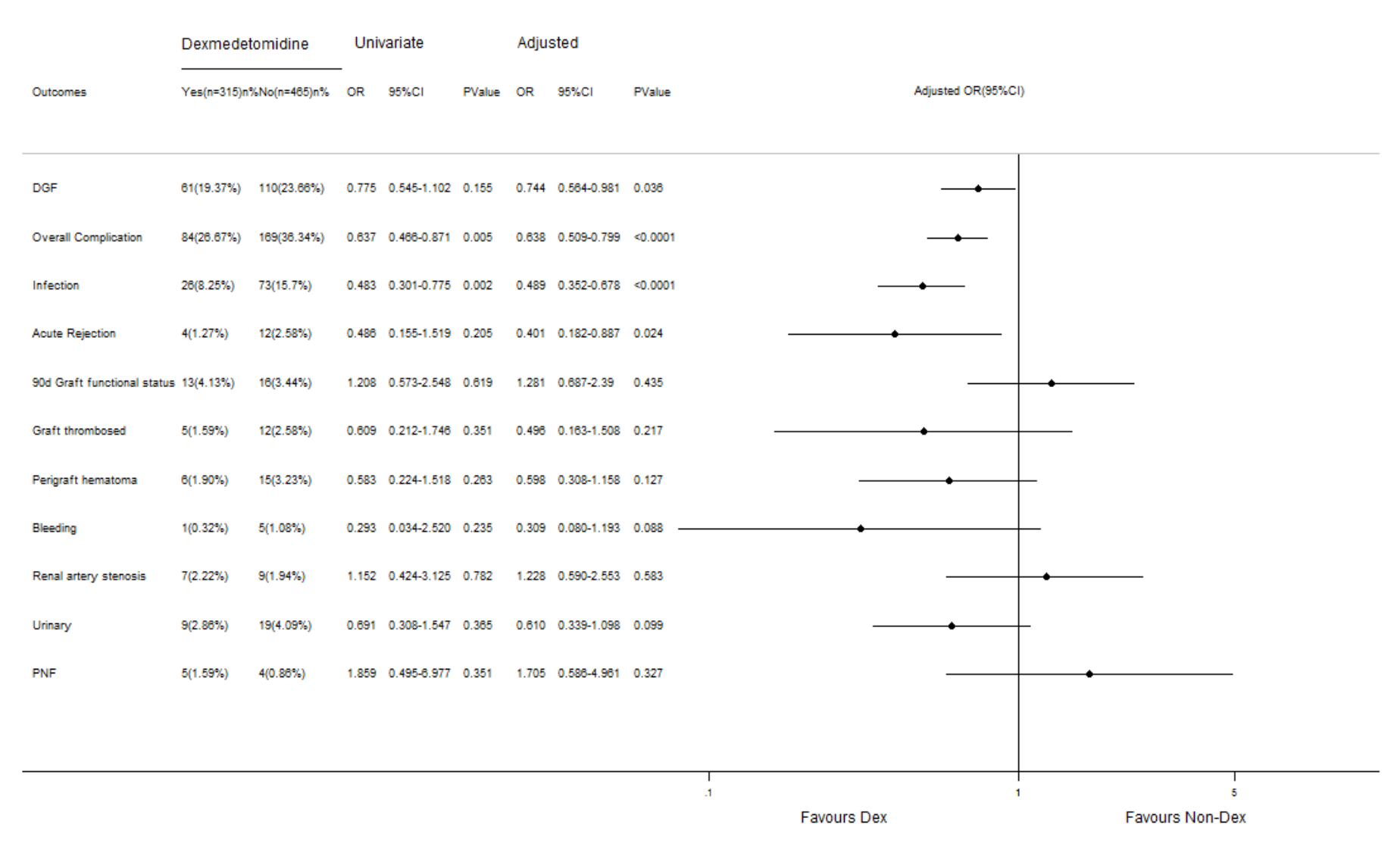

Figure 2: Effects of dexmedetomidine on post transplantation complications. Values are numbers (percent) for categorical variables; CI: confidence interval; OR: odds ratio; DGF: delayed graft function; PNF: Primary no function; DEX: dexmedetomidine.

In conclusion, perioperative use of dexmedetomidine was associated with a reduced incidence of DGF, infection, graft rejection, overall complications and LOS in renal transplantation patients. This improvement persisted after propensity weight and risk-adjustment.

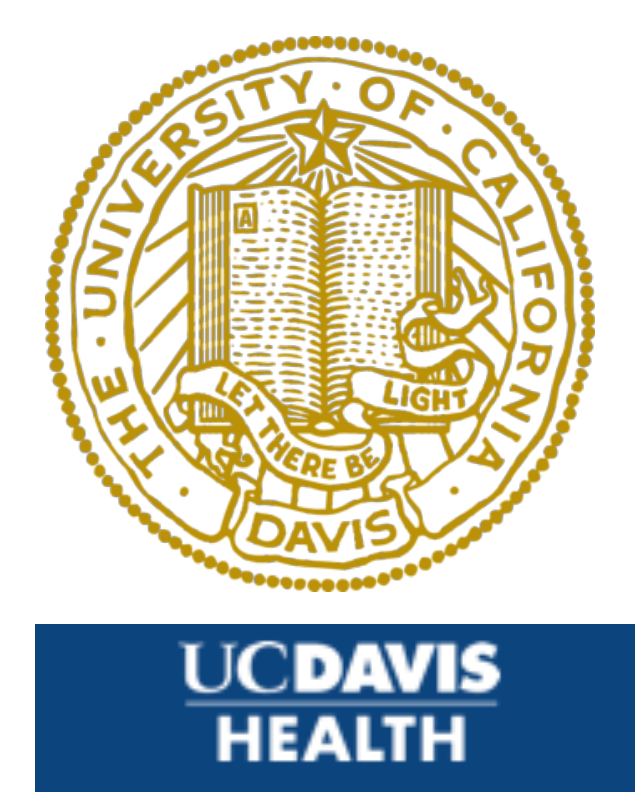

